\title{
QCD thermodynamics with continuum extrapolated Wilson fermions II.
}

\author{
Szabolcs Borsanyi $*$ Christian Holbling $\oplus$ and Balint C. Toth $\ddagger$ \\ University of Wuppertal, Department of Physics, Wuppertal D-42097, Germany \\ Stephan Durr \$ Stefan Krieg and Kalman K. Szabd** \\ University of Wuppertal, Department of Physics, Wuppertal D-42097, Germany and \\ Julich Supercomputing Center, Forschungszentrum Julich, Julich D-52425, Germany \\ Zoltan Fodor $\dagger$ \\ University of Wuppertal, Department of Physics, Wuppertal D-42097, Germany \\ Julich Supercomputing Center, Forschungszentrum Julich, Julich D-52425, Germany and \\ Eotvos University, Institute for Theoretical Physics, Budapest 1117, Hungary

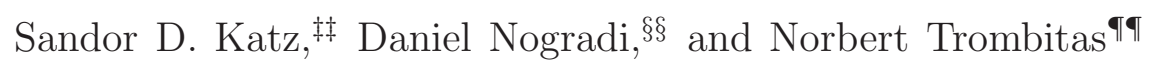 \\ Eotvos University, Institute for Theoretical Physics, Budapest 1117, Hungary and \\ MTA-ELTE Lendulet Lattice Gauge Theory Research Group, 1117 Budapest, Hungary

\begin{abstract}
We continue our investigation of $2+1$ flavor QCD thermodynamics using dynamical Wilson fermions in the fixed scale approach. Two additional pion masses, approximately $440 \mathrm{MeV}$ and $285 \mathrm{MeV}$, are added to our previous work at $545 \mathrm{MeV}$. The simulations were performed at 3 or 4 lattice spacings at each pion mass. The renormalized chiral condensate, strange quark number susceptibility and Polyakov loop is obtained as a function of the temperature and we observe a decrease in the light chiral pseudo-critical temperature as the pion mass is lowered while the pseudocritical temperature associated with the strange quark number susceptibility or the Polyakov loop is only mildly sensitive to the pion mass. These findings are in agreement with previous continuum results obtained in the staggered formulation.
\end{abstract}

\footnotetext{
*Electronic address: borsanyi@uni-wuppertal.de

${ }^{\dagger}$ Electronic address: hch@uni-wuppertal.de

${ }^{\ddagger}$ Electronic address: tothbalint@szofi.elte.hu
} 
$\S$ Electronic address: duerr@uni-wuppertal.de

『Electronic address: s.krieg@fz-juelich.de

**Electronic address: szaboka@general.elte.hu

${ }^{\dagger \dagger}$ Electronic address: fodor@bodri.elte.hu

${ }_{\ddagger}$ Electronic address: katz@bodri.elte.hu

$\S \S$ Electronic address: nogradi@bodri.elte.hu

$\mathbf{\top}^{\mathbb{\pi}}$ Electronic address: trombitas@bodri.elte.hu 


\section{INTRODUCTION}

The quantitative description of the quark gluon plasma (QGP) is in the focus of the heavy ion program at the accelerators RHIC (Brookhaven) and LHC (CERN). At the large energy densities achieved in these experiments quarks are no longer confined into detectable particles (hadrons) but form a nearly ideal fluid [1]. The QGP phase is separated from the hot gas of hadrons by a cross-over [2] at high enough collision energies. This transition leaves an imprint in the abundance of various particle species that are created at the breakup of the plasma [ $\left[\begin{array}{ll}3 & 6\end{array}\right]$ and the transition temperature can be modelled as a function of the collision energy or baryo-chemical potential [7-11].

Lattice simulations provide an excellent method to solve the underlying quantum field theory, Quantum Chromodynamics (QCD) in equilibrium [12]. Lattice calculations are valid and feasible both in the hadronic and in the quark gluon phase, allowing a first principles description of the transition itself. The appeal of lattices methods includes that no approximation is involved, the complete path integral of the discretized theory is calculated. The features of the continuum theory can then be obtained through continuum extrapolation from sufficiently high resolutions.

Contrary to experiments, lattice QCD has the advantage to access many possible theories with various quark masses [13]. E.g. it has been shown that QCD with infinite quark masses exhibits a 1st order transition between QGP and the confined phase [14], which is clearly signalled by the Polyakov loop, the exponentialized single quark free energy: the Polyakov loop is non-zero only for deconfined quarks. The first order nature persists when quarks become dynamical but heavy [15]. For very light quarks, the order of the transition depends on the number of light flavors. The transition is dominated by the restoration of chiral symmetry, signalled by the vanishing of the chiral condensate. For intermediate masses there is no real transition and both the Polyakov loop and the chiral condensate are approximate, remnant order parameters. In this work we will study these for several sets of quark masses, complemented by a measure of the confinement of the strange quarks, the strange quark number susceptibility.

Results with physical quark masses are abundant in the staggered formulation. The order of the transition is cross-over [2] with a chiral transition temperature $T_{c} \sim 155 \mathrm{MeV}$ [16 19]. The equation of state has been calculated with high precision [20 22], even at small but non- 
vanishing quark densities [23, 24] and there exist predictions for the freeze-out parameters [25-27] and fluctuations of various conserved charges [28, 29].

Yet it is not certain whether all systematics are controlled in staggered simulations. The staggered fermion action describes four flavors, which reduces to a single quark flavor by rooting the fermion sector, thus potentially giving up locality. This conceptually uncertain step is completely avoided in the Wilson formulation, which we also use in the present work. The theoretical soundness comes at a price. The Dirac spinor of Wilson fermions have four components, while the staggered spinor is a single complex field. More importantly chiral symmetry is explicitly broken at any lattice spacing, it is restored only in the continuum limit. This leads to a more complicated structure of divergences, and a demand for fine lattices. The use of a heavier than natural pion mass can significantly reduce the costs of an individual simulation. Indeed, although there exists zero temperature studies with Wilson fermions in the physical point [30], the description of the QCD transition with physical Wilson quarks is still missing.

There are also formulations which maintain a lattice version of chiral symmetry. These are computationally even more challenging than Wilson fermions. For thermodynamics results using overlap fermions see Ref. [31]. In the domain wall formulations physical quark masses have been recently reached, albeit not yet in continuum limit [32, 33].

The aim of thermodynamics studies with Wilson quarks goes beyond the obvious longterm goal of reaching the physical point. Several groups have already studied the chiral scaling with two flavors of Wilson quarks [34 39], and also in the twisted mass formulation [40, 41]. The transition temperature with infinitely heavy strange quarks monotonically decreases when the pion mass is lowered. Extrapolations to the physical pion mass give a value around $\sim 170 \mathrm{MeV}$ [36, 37, 40, 41]].

Most works with $2+1$ flavors of Wilson quarks address the phenomenology of QGP e.g. the equation of state [42] (using a pion mass of $m_{\pi} \sim 550 \mathrm{MeV}$ ). For some applications anisotropic lattices were introduced and the quark number susceptibilities [43] and transport coefficients [44, 45] have been calculated $\left(m_{\pi} \sim 392 \mathrm{MeV}\right)$. In [46, 47] we have started a study of $2+1$ flavor QCD thermodynamics using the Wilson fermion formulation with a fixed pion mass of $m_{\pi} \sim 545 \mathrm{MeV}$. A careful continuum extrapolation was performed and the results were found in agreement with the continuum extrapolated staggered simulations with equal pion mass. We have worked out the details of the renormalization procedure and 
in this work we reapply these for two new sets of quark masses. In this work we calculate three quantities of interest, the chiral condensate, strange quark number susceptibility and the Polyakov loop for 440 and $285 \mathrm{MeV}$ pions. A continuum extrapolation is performed for both masses.

Similarly to other $N_{f}=2$ studies we observe a monotonic shift in the chiral transition temperature as the physical point is approached. The pion mass dependence in the strange quark number susceptibility and the Polyakov loop is significantly milder.

The picture can only be complete if the temperature scans are shown together with data at the physical point. Such simulations with Wilson quarks are beyond our resources for now. Thus we use the continuum extrapolations from our staggered program to illustrate our expectations. This also shows that decreasing the pion mass further towards the physical point decreases the pseudo-critical temperature associated with the light chiral condensate whereas it does not substantially effect the pseudo-critical temperature associated with the strange quark number susceptibility.

The organization of the paper is as follows. In section [I] we summarize the simulation setup, parameters and algorithms that were used. In section [II the measured observables are given and their renormalization properties are discussed. In section IV we present the results of our investigations and we finally conclude in section $\mathrm{V}$.

\section{SIMULATION SETUP, LINE OF CONSTANT PHYSICS}

The Symanzik tree level improved action [48, 49] is used in the gauge sector while in the fermionic sector the clover [50] action further improved by six steps of stout smearing is adopted [51]. The clover coefficient is set to its tree level value $c_{S W}=1$ and the stout smearing parameter is chosen at $\varrho=0.11$. For more details see [52, 53] or [47] where the simulation setup was identical to the current work except for the values of the quark masses.

The light quarks $u$ and $d$ are assumed to be degenerate and a $2+1$ flavor algorithm is used. The HMC algorithm [54] is adopted for the light quarks and the RHMC algorithm [55] for the strange quark. Various algorithmic improvements are applied for speeding up the simulation: the Sexton-Weingarten multiple time scale integration [56], the Omelyan integration scheme [57] and even-odd preconditioning [58].

Finite temperature is introduced as a finite Euclidean temporal extent of the lattice. If 
the lattice is isotropic, i.e. the lattice spacing is identical in all directions, the temperature $T=1 / a N_{t}$ is set by the number of time slices $N_{t}$. Leaving the bare parameters unchanged one can thus vary the temperature by simulating at different values of $N_{t}$. To facilitate a continuum extrapolation three or four sets of bare parameters have to be determined, each corresponding to a different lattice spacing, but otherwise to the same physics (in terms of a selection of mass ratios). This is called the fixed-scale approach [59].

An alternative approach (mainly used in quenched and staggered simulations as well as in studies with exact chiral symmetry) keeps the number of time slices $\left(N_{t}\right)$ constant in a temperature scan and the lattice spacing is varied continuously through the bare parameters to tune the temperature.

In the absence of additive divergences in the bare parameters and assuming the feasibility of the interpolation of various counterterms the latter approach has a clear advantage: the simulation temperature can be selected without restriction. The somewhat low temperature resolution of the fixed-scale approach could be trivially improved by anisotropy, but also by redefining the temporal boundary conditions [60, 61].

In the Wilson formulation the additive divergences prevent the easy interpolation of zero temperature data. Repeated simulations at zero temperature are costly, (even more so if the action is anisotropic [62]) and one uses as few sets of bare parameters as possible, leading to the fixed scale approach, that we also use in this work in an isotropic setting. In many cases authors even forego the continuum extrapolation to spare the extra effort from the use of several parameter sets.

In this work we are working with four lattice spacings determined by the inverse gauge coupling $\beta$. As in Ref. [47] we use $\beta=3.30$, 3.57, 3.70 and 3.85 corresponding to lattice spacings from about $0.13 \mathrm{fm}$ to $0.05 \mathrm{fm}$. The scale was set by $m_{\Omega}=1672 \mathrm{MeV}$. The temperature at each fixed bare coupling $\beta$ is varied in discrete steps by varying $N_{t}$.

In our past work [47] the pion mass was relatively heavy, around $545 \mathrm{MeV}$. Two sets of simulations were performed in the current work each corresponding to a fixed $m_{\pi} / m_{\Omega}$ and $m_{K} / m_{\Omega}$ mass ratio. In the first set the quark masses were tuned to $m_{\pi} / m_{\Omega} \simeq 0.26$ and $m_{K} / m_{\Omega} \simeq 0.34$. These correspond to about $m_{\pi}=440 \mathrm{MeV}$ and $m_{K}=570 \mathrm{MeV}$. At this pion mass the simulations were performed at all 4 lattice spacings. Finite volume effects are expected to be small since $m_{\pi} L>7$ at each lattice spacing.

In the second set the meson masses were tuned to $m_{\pi} / m_{\Omega} \simeq 0.17$ and $m_{K} / m_{\Omega} \simeq 0.32$, 


\begin{tabular}{|c|c|c|c|c|}
\hline$\beta$ & $a m_{u d}$ & $a m_{s}$ & $N_{s}$ & $N_{t}$ \\
\hline \hline 3.30 & -0.1122 & -0.0710 & 32 & $6-16, \quad 32$ \\
\hline 3.57 & -0.0347 & -0.0115 & 48 & $6-16,64$ \\
\hline 3.70 & -0.0181 & 0.0 & 48 & $8-24,48$ \\
\hline 3.85 & -0.0100 & 0.0050 & 64 & $8-36,64$ \\
\hline \hline$\beta$ & $a m_{u d}$ & $a m_{s}$ & $N_{s}$ & $N_{t}$ \\
\hline \hline 3.30 & -0.1245 & -0.0710 & 32 & $6-16,32$ \\
\hline 3.57 & -0.0443 & -0.0115 & 48 & $8-24,64$ \\
\hline 3.70 & -0.0258 & 0.0 & 64 & $8-24,96$ \\
\hline
\end{tabular}

TABLE I: Bare parameters for the $440 \mathrm{MeV}$ pion mass (top) and the $285 \mathrm{MeV}$ pion mass (bottom) simulations. The $N_{t}$ values used for the finite temperature runs and the values used for the zero temperature runs are separated by a comma.

corresponding to about $m_{\pi}=285 \mathrm{MeV}$ and $m_{K}=525 \mathrm{MeV}$. At these pion masses the simulations were performed at 3 lattice spacings and for the finite volume of the system $m_{\pi} L>5.4$ holds.

At each lattice spacing, i.e. fixed $\beta$, the mass of the strange quark $m_{s}$ is fixed at its physical value across all three pion masses and the physical point would be approached by changing $m_{u d}$ only. Hence as $m_{u d}$ is lowered, both $m_{\pi}$ and $m_{K}$ decrease towards their physical values.

A summary of the various pion and kaon masses used in our past and current work is shown in figure 1. The bare quark masses, spatial and temporal lattice extents are shown in table 【 while the measured meson, baryon and PCAC masses are shown in table II. As can be seen $m_{\Omega}$ and hence the lattice spacing depends rather mildly on the light quark masses. At each finite temperature point around 1000-1500 equilibrated unit length trajectories were generated while we collected around 1000 trajectories at the zero temperature points. Autocorrelation times are around 5 - 25 trajectories close to the transition temperature depending on the quantity, lattice spacing and pion mass. 


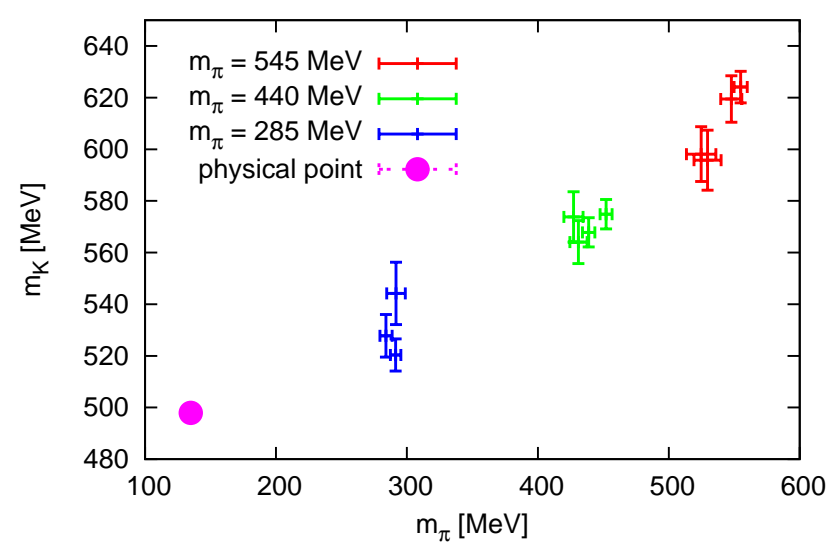

FIG. 1: The various pion and kaon masses used in our past and current work. The heaviest pion mass is from our past work [47], the 4 red data points correspond to 4 lattice spacings. For the $m_{\pi}=440 \mathrm{MeV}$ point also 4 lattice spacings are used, while for the lightest pion mass, $m_{\pi}=285$ $\mathrm{MeV}$ we have simulated at 3 lattice spacings. The physical point is also shown for comparison. The scale is set by $m_{\Omega}=1672 \mathrm{MeV}$.

\section{OBSERVABLES}

The temperature dependencies of three quantities are determined in the current work, the renormalized light chiral condensate, the strange quark number susceptibility and the renormalized Polyakov loop.

\section{A. Chiral condensate}

The bare light chiral condensate requires both additive and multiplicative renormalization. The details of the full renormalization procedure are given in [47] following the references [63, 64] and will be summarized below.

Additive renormalization at $T>0$ is implemented by the subtraction of $T=0$ quantities as this difference is free from polynomial divergences in the inverse of the lattice spacing. Multiplicative renormalization is then achieved by the multiplication of the PCAC mass $m_{P C A C}$ and the finite renormalization constant $Z_{A}$. The latter were determined in the chiral limit from 3-flavor simulations in [47] along the lines of [30, 65] and can be taken from there directly for each $\beta$. Finally the Ward identity establishes a relationship [64] between the 


\begin{tabular}{|c|c|c|c|c|c|}
\hline$\beta$ & $m_{\pi} / m_{\Omega}$ & $m_{K} / m_{\Omega}$ & $a m_{P C A C}$ & $a m_{\Omega}$ & $a[\mathrm{fm}]$ \\
\hline \hline 3.30 & $0.262(3)$ & $0.340(3)$ & $0.0248(2)$ & $1.11(1)$ & $0.133(1)$ \\
\hline 3.57 & $0.270(3)$ & $0.344(3)$ & $0.01710(5)$ & $0.737(7)$ & $0.088(1)$ \\
\hline 3.70 & $0.258(4)$ & $0.337(5)$ & $0.01266(3)$ & $0.578(8)$ & $0.069(1)$ \\
\hline 3.85 & $0.256(4)$ & $0.343(6)$ & $0.00890(1)$ & $0.446(7)$ & $0.053(1)$ \\
\hline \hline$\beta$ & $m_{\pi} / m_{\Omega}$ & $m_{K} / m_{\Omega}$ & $a m_{P C A C}$ & $a m_{\Omega}$ & $a[\mathrm{fm}]$ \\
\hline \hline 3.30 & $0.174(4)$ & $0.325(7)$ & $0.0084(2)$ & $0.97(2)$ & $0.117(3)$ \\
\hline 3.57 & $0.174(2)$ & $0.311(4)$ & $0.00693(4)$ & $0.723(8)$ & $0.087(1)$ \\
\hline 3.70 & $0.170(1)$ & $0.316(5)$ & $0.00481(2)$ & $0.560(9)$ & $0.067(1)$ \\
\hline
\end{tabular}

TABLE II: Spectroscopy and physical scale results from zero temperature simulations, top: $m_{\pi}=$ $440 \mathrm{MeV}$, bottom: $m_{\pi}=285 \mathrm{MeV}$. The lattice spacings are set by $m_{\Omega}=1672 \mathrm{MeV}$.

chiral condensate and the integrated pion correlator leading to the final expression for the fully renormalized condensate at finite temperature,

$$
m_{R}\langle\bar{\psi} \psi\rangle_{R}(T)=2 N_{f} m_{P C A C}^{2} Z_{A}^{2} \Delta_{P P}(T)
$$

where,

$$
\Delta_{P P}(T)=\int d^{4} x\left\langle P_{0}(x) P_{0}(0)\right\rangle(T)-\int d^{4} x\left\langle P_{0}(x) P_{0}(0)\right\rangle(T=0)
$$

where $P_{0}(x)$ is the bare pseudo-scalar density; for more details see [47]. The final result in [47] was shown for $m_{R}\langle\bar{\psi} \psi\rangle_{R}(T) / m_{\pi}^{4}$ since this combination is dimensionless. However when comparing different pion masses as in the current work this normalization is not convenient because it introduces an artificial pion mass dependence through the $4^{\text {th }}$ power. It turns out that the normalization $m_{R}\langle\bar{\psi} \psi\rangle_{R}(T) / m_{\pi}^{2} / m_{\Omega}^{2}$ is more suitable. This is because according to the GMOR relation at $T=0$, the quark mass times the chiral condensate is proportional to $m_{\pi}^{2}$ to lowest order in chiral perturbation theory. All results related to the chiral condensate will be presented with the latter normalization and also the final result in [47] will be converted into it for comparison. 


\section{B. Strange quark number susceptibility}

The strange quark number susceptibility $\chi_{s}=T / V \partial^{2} \log Z / \partial \mu_{s}^{2}$, where $\mu_{s}$ is the strange quark chemical potential, can be made dimensionless by considering $\chi_{s} / T^{2}$ and can be improved at tree level by the division of its infinite volume and massless Stefan-Boltzmann limit at each finite $N_{t}$. The Stefan-Boltzmann values for each $N_{t}$ were listed in [47]. Furthermore $\chi_{s} / T^{2}$ is a finite quantity in the continuum hence does not require any further renormalization factors. The strange quark number susceptibility is sensitive to the confinementdeconfinement temperature of the strange quark and as we will see is only mildly dependent on the pion mass.

\section{Polyakov loop}

In order to renormalize the Polyakov loop one may use zero temperature quantities sim-

ilarly to our description of the renormalized chiral condensate [16]. However it is more convenient and less noisy to only use the finite temperature Polyakov loop itself [66].

Our renormalization procedure for the Polyakov loop follows [47]. The additive divergence of the free energy can be removed by the following renormalization prescription: a fixed value $L_{*}$ can be fixed for the renormalized Polyakov loop at a fixed but arbitrary temperature $T_{*}>T_{c}$. This prescription leads to the following renormalized Polyakov loop $L_{R}$ in terms of the bare quantity $L_{0}$,

$$
L_{R}(T)=\left(\frac{L_{*}}{L_{0}\left(T_{*}\right)}\right)^{\frac{T_{*}}{T}} L_{0}(T) .
$$

We choose $T_{*}=0.143 m_{\Omega}$ and $L_{*}=1.2$ similarly to [47] while other choices would simply correspond to other renormalization schemes. For instance one may fix $T_{*}$ in units of $T_{c}$ as well.

\section{RESULTS AND CONTINUUM LIMIT}

At the $m_{\pi}=440 \mathrm{MeV}$ point the simulations were performed at 4 lattice spacings while at the $m_{\pi}=285 \mathrm{MeV}$ point only at 3 . Since the fixed scale approach is used where the temperature can only be changed by discrete amounts corresponding to the discrete changes 

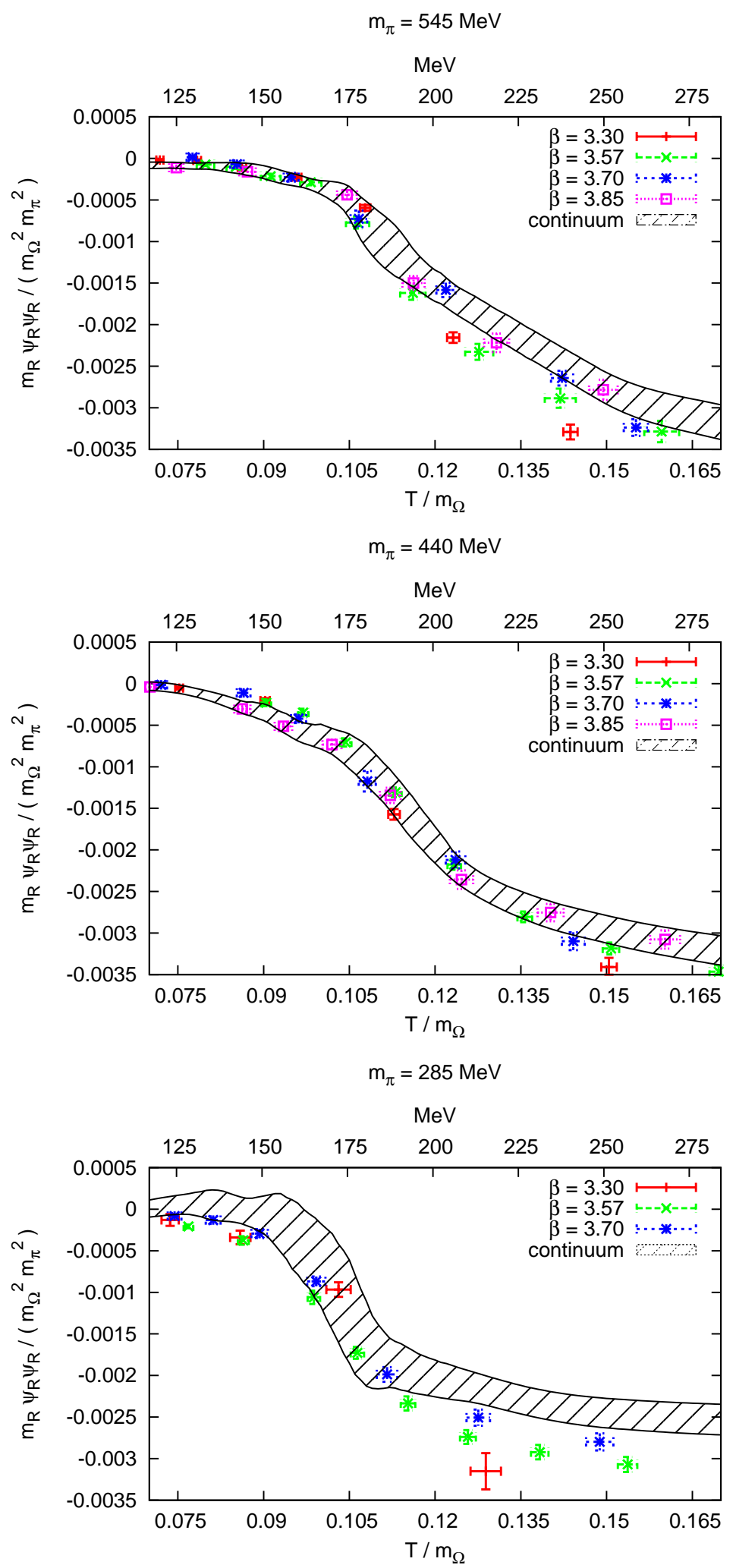

FIG. 2: The renormalized chiral condensate for $m_{\pi}=545 \mathrm{MeV}$ (top, from [47]), $m_{\pi}=440 \mathrm{MeV}$ (middle) and $m_{\pi}=285 \mathrm{MeV}$ (bottom). The continuum extrapolated results are also shown by the solid band. 

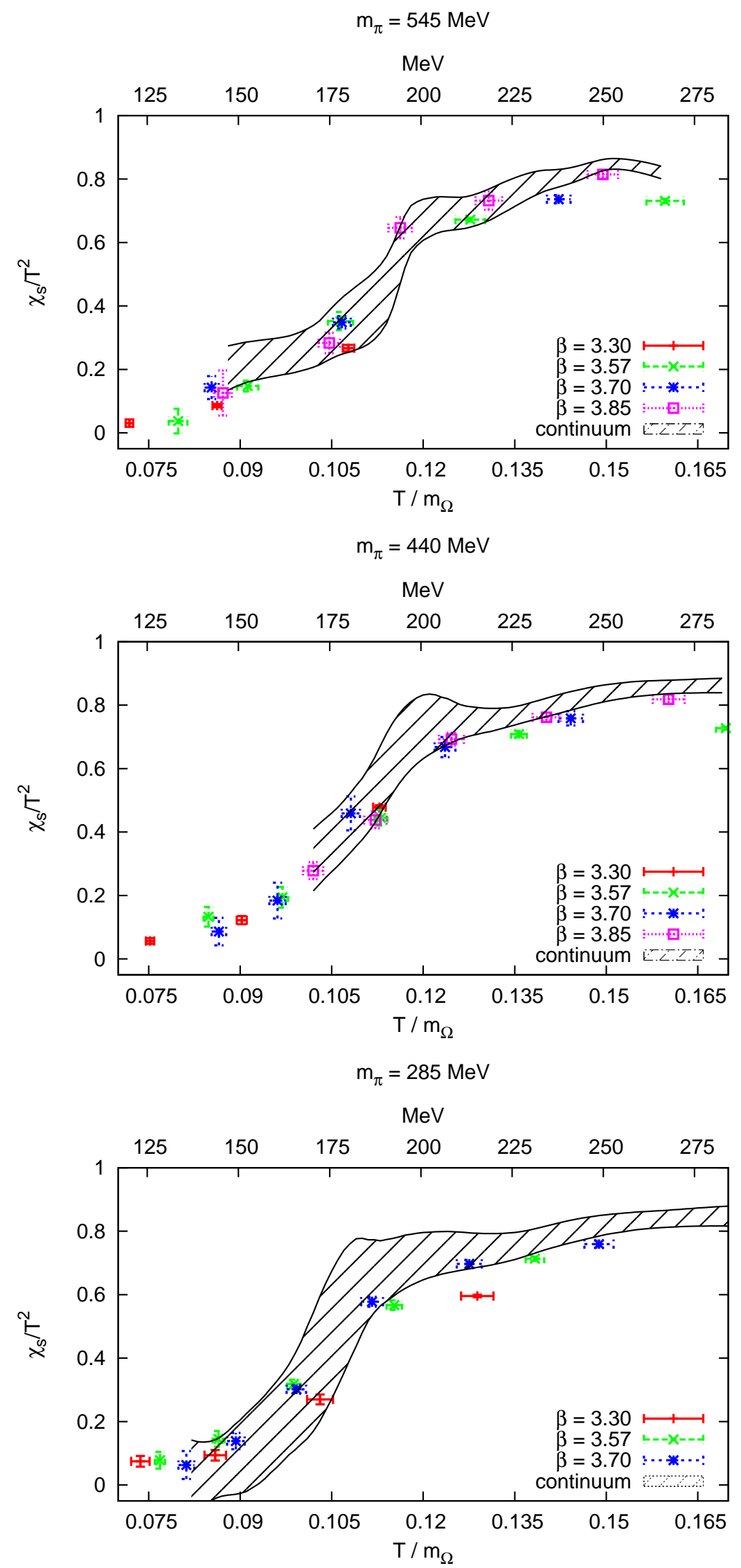

FIG. 3: The strange quark number susceptibility for $m_{\pi}=545 \mathrm{MeV}$ (top, from [47]), $m_{\pi}=440$ $\mathrm{MeV}$ (middle) and $m_{\pi}=285 \mathrm{MeV}$ (bottom). The continuum extrapolated results are also shown 
in $N_{t}$ at each bare coupling $\beta$ an interpolation is necessary in order to have a continuous curve as a function of temperature for each observable. In our previous work in [47] a spline interpolation was adopted and in the current work we add another method.

The previous method of [47] consists of randomly placing node points for cubic spline interpolations and the parameters of the spline are continuum extrapolated assuming $O(a)$ and $O\left(a^{2}\right)$ cut-off effects. Each result corresponding to a fixed set of node points is weighted by its fit quality. The deviation of the two continuum extrapolations as well as the spread with the various random choices for the nodes allow us to estimate systematic effects coming from both the continuum extrapolation and the interpolation. For more details see [47].

In the current work we analyzed all of our data using a second method as well. Clearly the expectation is that all quantities are monotonous functions of the temperature. This constraint is imposed on our cubic spline fit following the algorithm [67]. Naturally the statistical uncertainty of our measured data points will lead to a statistical uncertainty for the interpolated curve. The obtained continuous interpolated curves and their error for each $\beta$ can then be used for a continuum extrapolation at each $T$ (note that in the previous method the interpolation and extrapolation was performed simultaneously in a global fit). We have performed the continuum extrapolation assuming both $O(a)$ an $O\left(a^{2}\right)$ cut-off effects but the $\chi^{2} /$ dof values of the $O\left(a^{2}\right)$ were much better hence completely dominate the final result after performing an AIC weighted averaging [68 71]. The data from [47] is reanalized in this slightly different way in order to have a consistent analysis for all 3 pion masses and it is reassuring to see that the final continuum results agree with the previous analysis in [47].

In order to assess the size of systematic uncertainties we perform all continuum fits by keeping all 4 lattice spacings for the 2 heavier pion masses and also by dropping the roughest one, $\beta=3.30$ and hence using only 3 . The two continuum results are then weighted according to [68 71]. The deviation between the two continuum results is taken into account as a systematic uncertainty.

At the lightest pion mass $m_{\pi}=285 \mathrm{MeV}$ we only have data for $\beta=3.30,3.57$ and 3.70, i.e. the finest lattice spacing corresponding to $\beta=3.85$ was beyond reach. Unfortunately in this case it turned out that the $\beta=3.30$ lattice spacing could not be used for the continuum extrapolation of the chiral condensate and strange quark number susceptibility because the resulting fits had bad $\chi^{2} /$ dof values. Hence at this lightest pion mass point only 2 lattice 

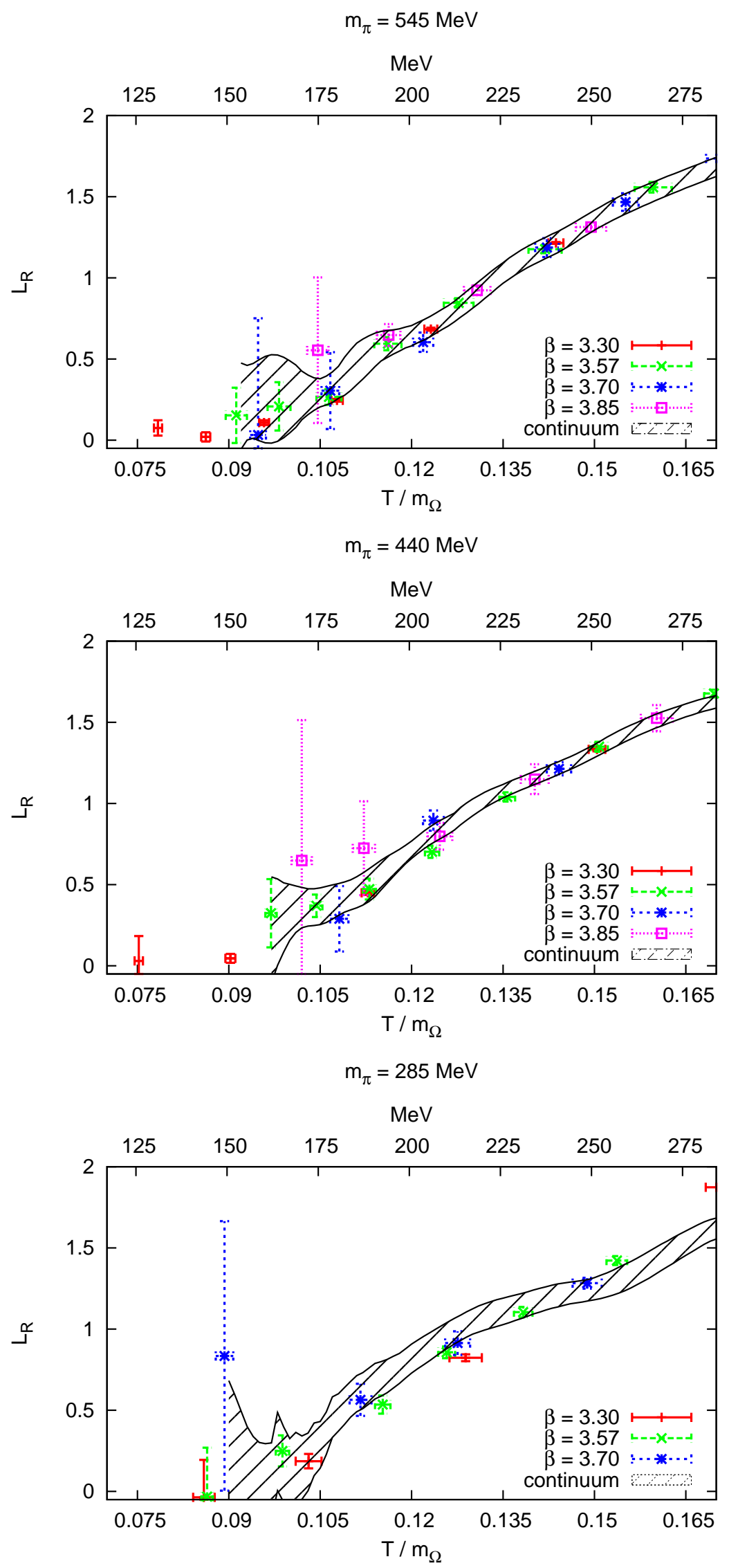

FIG. 4: The renormalized Polyakov loop for $m_{4^{\pi}}=545 \mathrm{MeV}$ (top, from [47]), $m_{\pi}=440 \mathrm{MeV}$ (middle) and $m_{\pi}=285 \mathrm{MeV}$ (bottom) pion masses. The continuum extrapolated results are also 
spacings are used, $\beta=3.57$ and $\beta=3.70$ which of course leads to continuum fits where the number of data points equals the number of parameters. For this reason our continuum results for the lightest pion is not fully under control and we call them continuum estimates only. As we will see from the continuum extrapolation of the Polyakov loop cut-off effects are very small in this quantity and all 3 lattice spacings can be used for $m_{\pi}=285 \mathrm{MeV}$. Hence the continuum result for the renormalized Polyakov loop is fully under control.

In order to check the robustness of our results we have reanalyzed the two new data sets corresponding to $m_{\pi}=440 \mathrm{MeV}$ and $285 \mathrm{MeV}$ using the strategy in [47]. There the systematic uncertainty was quantified by considering randomly chosen nodes for the spline interpolation as well as performing $O(a)$ and $O\left(a^{2}\right)$ fits to the continuum simultaneously. Comparison of the two methods for all 3 cases is again reassuring and shows that the statistical and systematic effects have been estimated correctly.

On all figures below the results from the second interpolation/extrapolation method is used. Note that even though monotonous interpolations are used and the continuum extrapolated central values are also monotonous, the errors on the central values are temperature dependent and may lead to a non-monotonous error band. This does happen in some cases.

The renormalized light chiral condensate is shown in figure 2 for all 3 pion masses and the strange quark number susceptibility is shown in figure 3 again for all three pion masses while the renormalized Polyakov loop is shown in figure 4, In each case the solid band shows the result of our continuum extrapolations.

Once continuum results are obtained at each of the three pion masses these continuum results can be compared for each observable. Clearly, the pseudo-critical temperature defined by the chiral condensate is decreasing with decreasing pion mass, see figure 5 . The pseudocritical temperatures corresponding to the strange quark number susceptibility and Polyakov loop on the other hand are only mildly sensitive, if at all, to the pion masses, see figure 6 and 7. On these comparison plots we also show the result of past investigations using the staggered formulation where continuum extrapolated results were possible to obtain at the physical pion mass [18].

Clearly, the staggered physical and continuum results fit nicely into the trend observed for the Wilson results: the pseudo-critical temperature corresponding to the light chiral condensate is decreasing further with decreasing pion mass while the strange quark number susceptibility shows only mild or no dependence. This is presumably because the strange 
quark in the valence sector is the one dominating the strange quark number susceptibility and the light quarks enter only through their sea contribution. On the other hand the light chiral condensate depends on the pion mass through both the sea and valence sectors.

\section{SUMMARY AND OUTLOOK}

In this paper we continued our program of lattice QCD thermodynamics using the Wilson fermion formulation. Our previously published results at a relatively heavy pion mass $m_{\pi}=$ $545 \mathrm{MeV}$ was extended by including two ligther pions, $m_{\pi}=440 \mathrm{MeV}$ and $m_{\pi}=285 \mathrm{MeV}$. Our main goal was to investigate the pion mass dependence of several observables which may be used to define a pseudo-critical temperature. The continuum extrapolation was fully under control for $m_{\pi}=440 \mathrm{MeV}$ but since we only used two lattice spacings for $m_{\pi}=285$ $\mathrm{MeV}$, in the latter case we refer to it as a continuum estimate only (except for the Polyakov loop where three lattice spacings were used and the result is hence fully under control). In any case the continuum results support the picture that emerged from staggered simulations: the pseudo-critical temperature associated with the light quarks is much more sensitive to the pion mass than the pseudo-critical temperature associated with the strange quark. The light chiral condensate may be used to define the former and the strange quark number susceptibility may be used to define the latter. The Polyakov loop which becomes an order parameter in the infinitely massive quark limit also shows little pion mass dependence.

We see a clear decrease in $T_{c}$ obtained from the light chiral condensate as the pion mass decreases and not much sensitivity to the pion mass in $T_{c}$ obtained from the strange quark. The physical pion mass is beyond reach for our simulations with Wilson fermions however with staggered fermions these are readily available. The comparison of our 3 Wilson continuum results corresponding to $m_{\pi}=545,440$ and $285 \mathrm{MeV}$ with the staggered continuum result at physical pions confirm this picture further as the light chiral condensate curve as a function of temperature moves further to the left as $m_{\pi}=285 \mathrm{MeV}$ decreases to the physical point. However the strange quark number susceptibility as a function of temperature is only mildly sensitive to the pion mass.

One may attempt to extrapolate the chiral condensate to the physical point but judging from figure 5 the result will have a rather large uncertainty and therefore will not be very

informative. In order to obtain results with reasonable accuracy at the physical point a 


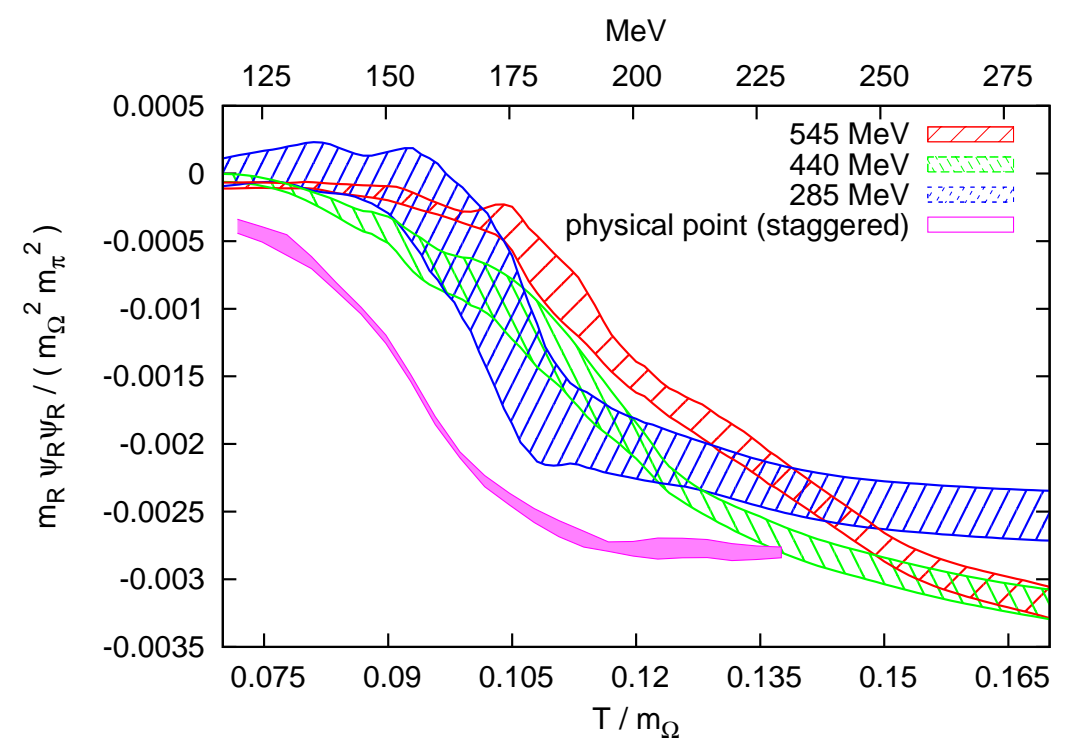

FIG. 5: Comparison of the continuum renormalized chiral condensate results for the three pion masses $m_{\pi}=545 \mathrm{MeV}, m_{\pi}=440 \mathrm{MeV}$ and $m_{\pi}=285 \mathrm{MeV}$. The continuum result at the physical point is also shown, obtained from staggered simulations. A downward shift in the pseudo-critical temperature with decreasing pion masses is clearly visible.

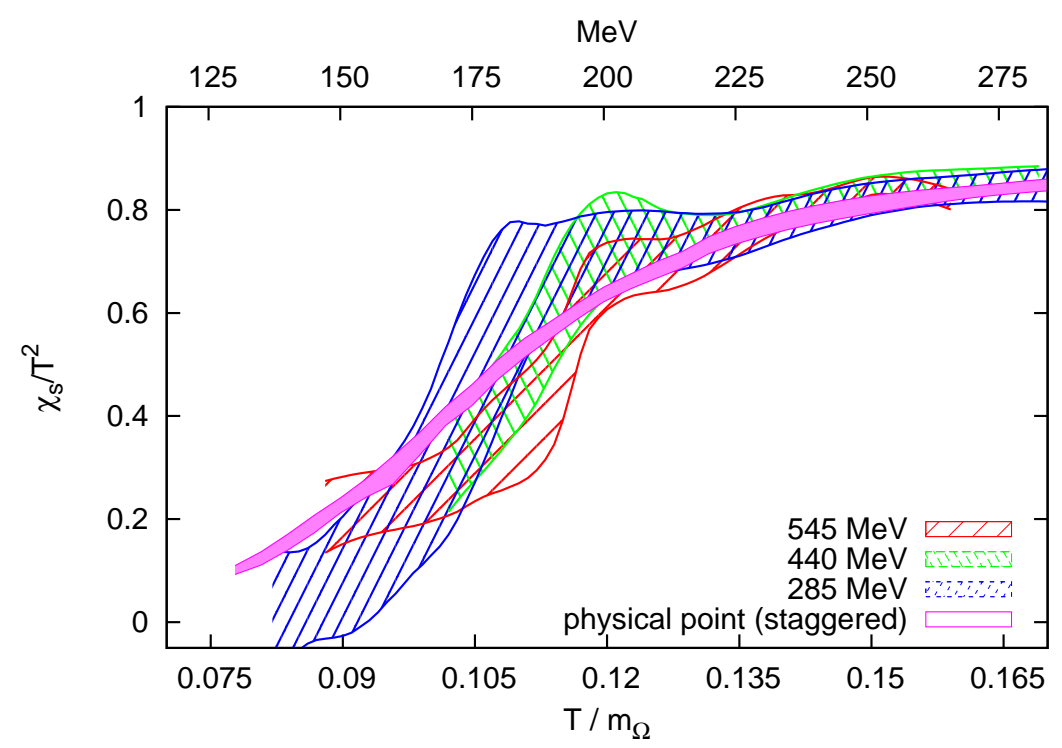

FIG. 6: Comparison of the continuum strange quark number susceptibility results for the three pion masses $m_{\pi}=545 \mathrm{MeV}, m_{\pi}=440 \mathrm{MeV}$ and $m_{\pi}=285 \mathrm{MeV}$. The continuum result at the physical point is also shown, obtained from staggered simulations. 


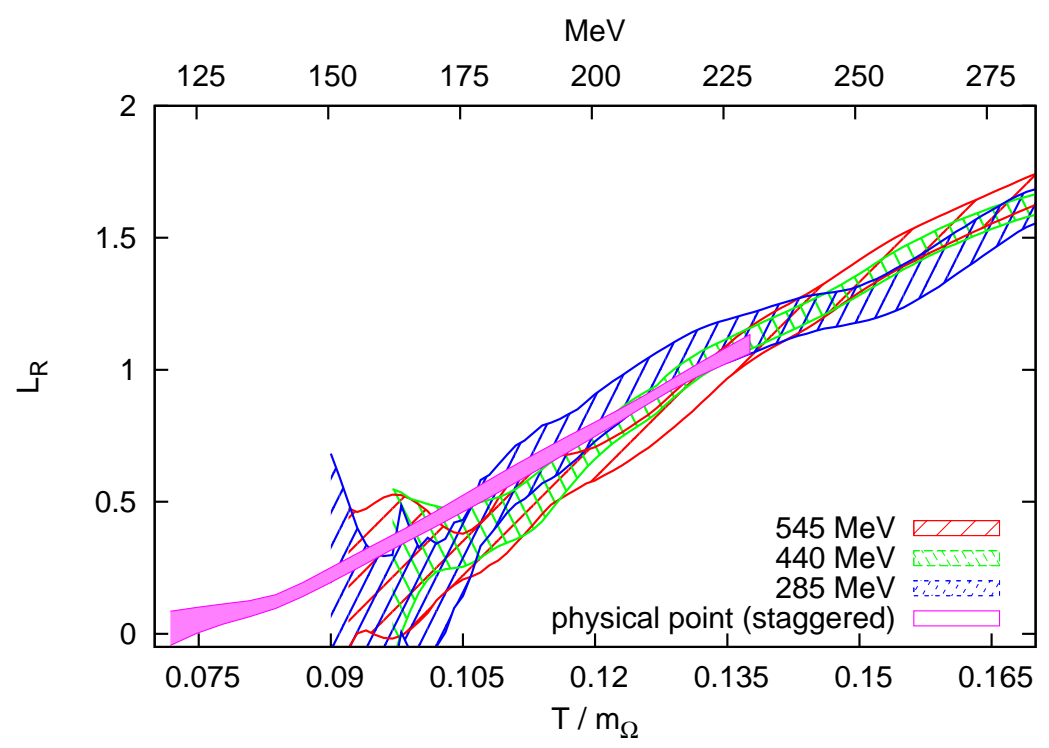

FIG. 7: Comparison of the continuum renormalized Polyakov loop results for the three pion masses $m_{\pi}=545 \mathrm{MeV}, m_{\pi}=440 \mathrm{MeV}$ and $m_{\pi}=285 \mathrm{MeV}$. The continuum result at the physical point is also shown, obtained from staggered simulations.

simulation at at least one lattice spacing directly at the physical point will be needed.

\section{Acknowledgment}

Computations were carried out on both GPU clusters [72] at the University of Wuppertal, Germany and Eotvos University, Budapest, Hungary and also on the BG/Q supercomputer in Forschungszentrum Juelich, Germany.

This work was supported by the EU Framework Programme 7 grant (FP7/20072013)/ERC No 208740, by the Deutsche Forschungsgemeinschaft grant SFB-TR 55 and by the grant OTKA-NF-104034 by OTKA.

[1] L. Kumar, Mod.Phys.Lett. A28 (2013) 1330033, [arXiv:1311.3426].

[2] Y. Aoki, G. Endrodi, Z. Fodor, S. Katz, and K. Szabo, Nature 443 (2006) 675-678, [heplat/0611014].

[3] P. Braun-Munzinger, K. Redlich, and J. Stachel, nucl-th/0304013. 
[4] J. Rafelski, J. Letessier, and G. Torrieri, Phys.Rev. C72 (2005) 024905, [nucl-th/0412072].

[5] A. Andronic, P. Braun-Munzinger, and J. Stachel, Nucl.Phys. A772 (2006) 167-199, [nuclth/0511071].

[6] J. Cleymans, H. Oeschler, K. Redlich, and S. Wheaton, Phys.Rev. C73 (2006) 034905, [hep$\mathrm{ph} / 0511094]$.

[7] P. Braun-Munzinger, J. Stachel, and C. Wetterich, Phys.Lett. B596 (2004) 61-69, [nuclth/0311005].

[8] A. Andronic, P. Braun-Munzinger, and J. Stachel, Phys.Lett. B673 (2009) 142-145, [arXiv:0812.1186].

[9] STAR Collaboration, B. Abelev et al., Phys.Rev. C79 (2009) 034909, [arXiv:0808.2041].

[10] F. Becattini, M. Bleicher, T. Kollegger, T. Schuster, J. Steinheimer, et al., Phys.Rev.Lett. 111 (2013) 082302, [arXiv:1212.2431].

[11] F. Becattini, E. Grossi, M. Bleicher, J. Steinheimer, and R. Stock, Phys.Rev. C90 (2014), no. 5 054907, [arXiv:1405.0710].

[12] P. Petreczky, J.Phys. G39 (2012) 093002, [arXiv:1203.5320].

[13] F. R. Brown, F. P. Butler, H. Chen, N. H. Christ, Z.-h. Dong, et al., Phys.Rev.Lett. 65 (1990) $2491-2494$.

[14] T. Celik, J. Engels, and H. Satz, Phys.Lett. B125 (1983) 411.

[15] P. Hasenfratz, F. Karsch, and I. Stamatescu, Phys.Lett. B133 (1983) 221.

[16] Y. Aoki, Z. Fodor, S. Katz, and K. Szabo, Phys.Lett. B643 (2006) 46-54, [hep-lat/0609068].

[17] Y. Aoki, S. Borsanyi, S. Durr, Z. Fodor, S. D. Katz, et al., JHEP 0906 (2009) 088, [ arXiv:0903.4155.

[18] Wuppertal-Budapest Collaboration, S. Borsanyi et al., JHEP 1009 (2010) 073, [ arXiv:1005.3508.

[19] A. Bazavov, T. Bhattacharya, M. Cheng, C. DeTar, H. Ding, et al., Phys.Rev. D85 (2012) 054503, [arXiv:1111.1710].

[20] S. Borsanyi, G. Endrodi, Z. Fodor, A. Jakovac, S. D. Katz, et al., JHEP 1011 (2010) 077, [ arXiv:1007.2580.

[21] S. Borsanyi, Z. Fodor, C. Hoelbling, S. D. Katz, S. Krieg, et al., Phys.Lett. B730 (2014) 99-104, [arXiv:1309.5258].

[22] HotQCD Collaboration, A. Bazavov et al., Phys.Rev. D90 (2014), no. 9 094503, [ 
arXiv:1407.6387.

[23] S. Borsanyi, G. Endrodi, Z. Fodor, S. Katz, S. Krieg, et al., JHEP 1208 (2012) 053, [ arXiv:1204.6710.

[24] BNLBielefeldCCNU Collaboration, P. Hegde, Nucl.Phys. A931 (2014) 851-855, [arXiv:1408.6305].

[25] A. Bazavov, H. Ding, P. Hegde, O. Kaczmarek, F. Karsch, et al., Phys.Rev.Lett. 109 (2012) 192302, [ arXiv:1208.1220].

[26] S. Borsanyi, Z. Fodor, S. Katz, S. Krieg, C. Ratti, et al., Phys.Rev.Lett. 111 (2013) 062005, [arXiv:1305.5161].

[27] S. Borsanyi, Z. Fodor, S. Katz, S. Krieg, C. Ratti, et al., Phys.Rev.Lett. 113 (2014) 052301, [arXiv:1403.4576].

[28] S. Borsanyi, Z. Fodor, S. D. Katz, S. Krieg, C. Ratti, et al., JHEP 1201 (2012) 138, [ arXiv:1112.4416.

[29] HotQCD Collaboration, A. Bazavov et al., Phys.Rev. D86 (2012) 034509, [arXiv:1203.0784].

[30] S. Durr, Z. Fodor, C. Hoelbling, S. Katz, S. Krieg, et al., Phys.Lett. B701 (2011) 265-268, [arXiv:1011.2403].

[31] S. Borsanyi, Y. Delgado, S. Durr, Z. Fodor, S. D. Katz, et al., Phys.Lett. B713 (2012) 342-346, [arXiv:1204.4089].

[32] M. I. Buchoff, M. Cheng, N. H. Christ, H. T. Ding, C. Jung, et al., Phys.Rev. D89 (2014), no. 5 054514, [arXiv:1309.4149].

[33] T. Bhattacharya, M. I. Buchoff, N. H. Christ, H.-T. Ding, R. Gupta, et al., Phys.Rev.Lett. 113 (2014), no. 8 082001, [arXiv:1402.5175].

[34] CP-PACS Collaboration Collaboration, A. Ali Khan et al., Phys.Rev. D63 (2001) 034502, [hep-lat/0008011].

[35] V. Bornyakov, R. Horsley, S. Morozov, Y. Nakamura, M. Polikarpov, et al., Phys.Rev. D82 (2010) 014504, [arXiv:0910.2392].

[36] V. Bornyakov, R. Horsley, Y. Nakamura, M. Polikarpov, P. Rakow, et al., PoS Lattice2010 (2014) 170, [arXiv:1102.4461].

[37] WHOT-QCD Collaboration, S. Ejiri et al., Phys.Rev. D82 (2010) 014508, [arXiv:0909.2121].

[38] B. B. Brandt, A. Francis, H. B. Meyer, and H. Wittig, JHEP 1303 (2013) 100, [ arXiv:1212.4200. 
[39] B. B. Brandt, A. Francis, H. B. Meyer, O. Philipsen, and H. Wittig, PoS LATTICE2013 (2014) 162, [arXiv:1310.8326].

[40] tmfT Collaboration, F. Burger et al., Phys.Rev. D87 (2013), no. 7 074508, [arXiv:1102.4530].

[41] F. Burger et al. [tmfT Collaboration], Phys. Rev. D 91, no. 7, 074504 (2015) arXiv:1412.6748 [hep-lat]].

[42] WHOT-QCD Collaboration Collaboration, T. Umeda et al., Phys.Rev. D85 (2012) 094508, [arXiv:1202.4719].

[43] P. Giudice, G. Aarts, C. Allton, A. Amato, S. Hands, et al., PoS LATTICE2013 (2014) 492, [arXiv:1309.6253].

[44] A. Amato, G. Aarts, C. Allton, P. Giudice, S. Hands, et al., Phys.Rev.Lett. 111 (2013), no. 17 172001, [ arXiv:1307.6763].

[45] G. Aarts, C. Allton, A. Amato, P. Giudice, S. Hands, et al., JHEP 1502 (2015) 186, [arXiv:1412.6411].

[46] S. Borsanyi, Z. Fodor, C. Hoelbling, S. D. Katz, S. Krieg, et al., PoS LATTICE2011 (2011) 209, [arXiv:1111.3500].

[47] S. Borsanyi, S. Durr, Z. Fodor, C. Hoelbling, S. D. Katz, et al., JHEP 1208 (2012) 126, [arXiv:1205.0440].

[48] K. Symanzik, Nucl.Phys. B226 (1983) 187.

[49] M. Luscher and P. Weisz, Commun.Math.Phys. 97 (1985) 59.

[50] B. Sheikholeslami and R. Wohlert, Nucl.Phys. B259 (1985) 572.

[51] C. Morningstar and M. J. Peardon, Phys.Rev. D69 (2004) 054501, [hep-lat/0311018].

[52] S. Capitani, S. Durr, and C. Hoelbling, JHEP 0611 (2006) 028, [ hep-lat/0607006].

[53] S. Durr, Z. Fodor, C. Hoelbling, R. Hoffmann, S. Katz, et al., Phys.Rev. D79 (2009) 014501, [arXiv:0802.2706].

[54] S. Duane, A. Kennedy, B. Pendleton, and D. Roweth, Phys.Lett. B195 (1987) 216-222.

[55] M. Clark and A. Kennedy, Phys.Rev.Lett. 98 (2007) 051601, [ hep-lat/0608015].

[56] J. Sexton and D. Weingarten, Nucl.Phys. B380 (1992) 665-678.

[57] T. Takaishi and P. de Forcrand, Phys.Rev. E73 (2006) 036706, [ hep-lat/0505020].

[58] T. A. DeGrand, Comput.Phys.Commun. 52 (1988) 161-164.

[59] T. Umeda, S. Ejiri, S. Aoki, T. Hatsuda, K. Kanaya, et al., Phys.Rev. D79 (2009) 051501, [arXiv:0809.2842]. 
[60] L. Giusti and H. B. Meyer, Phys.Rev.Lett. 106 (2011) 131601, [arXiv:1011.2727].

[61] T. Umeda, Phys.Rev. D90 (2014), no. 5 054511, [arXiv:1408.2328].

[62] R. G. Edwards, B. Joo, and H.-W. Lin, Phys.Rev. D78 (2008) 054501, [arXiv:0803.3960].

[63] M. Bochicchio, L. Maiani, G. Martinelli, G. C. Rossi, and M. Testa, Nucl.Phys. B262 (1985) 331.

[64] L. Giusti, F. Rapuano, M. Talevi, and A. Vladikas, Nucl.Phys. B538 (1999) 249-277, [heplat/9807014].

[65] S. Durr, Z. Fodor, C. Hoelbling, S. Katz, S. Krieg, et al., JHEP 1108 (2011) 148, [arXiv:1011.2711].

[66] S. Gupta, K. Huebner, and O. Kaczmarek, Phys.Rev. D77 (2008) 034503, [arXiv:0711.2251].

[67] F. N. Fritsch and R. E. Carlson, SIAM J. Numer. Anal. 17 (1980), no. 2 238-246.

[68] S. Konishi and G. Kitagawa, Information Criteria and Statistical Modeling. Springer Series in Statistics. Springer, 2008.

[69] G. Kitagawa and W. Gersch, Smoothness Priors Analysis of Time Series. Lecture Notes in Statistics 116. Springer New York, 1996.

[70] H. Akaike IEEE Trans. Automat. Contr. 19 (1974) 716723.

[71] H. Akaike Statistician 27 (1978) 217.

[72] G. I. Egri, Z. Fodor, C. Hoelbling, S. D. Katz, D. Nogradi, et al., Comput.Phys.Commun. 177 (2007) 631-639, [hep-lat/0611022]. 